\title{
EMPIRICAL INVESTIGATIONS OF CONCEPTUAL MODELING AND THE MODELING PROCESS
}

\author{
Wang Wang \\ Roger J. Brooks \\ Department of Management Science \\ Lancaster University Management School \\ Lancaster, LA1 4YX, U.K.
}

\begin{abstract}
Conceptual modeling, deciding what to include in the model, is a very important task in the modeling process. However, it has so far received relatively little attention in the literature and there is a lack of empirical data. This paper describes three empirical studies on conceptual modeling and the modeling process. In the first one, data on the time spent on different topics during real simulation projects by an expert and by groups of novice modelers was collected and analyzed. The second study was a questionnaire survey to obtain data on conceptual models and modeling processes for projects carried out by experienced modelers. The third study was an experiment to investigate the effect of model size on the ease of understanding of the model.
\end{abstract}

\section{INTRODUCTION}

Of all the tasks involved in a modeling project, conceptual modeling is probably the one that has received the least attention and consequently is the least well understood. Most other tasks such as data analysis, model building, verification and validation, and output analysis have a strong element of mathematics, statistics or logic. This has enabled techniques from other disciplines to be applied so that there are now well-established methods for most typical situations (e.g., see Law 2007). The nature of conceptual modeling is quite different, so much so that it is often described as being an art rather than a science (e.g., Shannon 1975). Most textbooks devote only a few pages to conceptual modeling and provide only a few general guidelines, with one notable exception being Robinson (2004) which includes two chapters on the topic.

The term conceptual modeling itself can cause confusion because of its different uses in different areas of science and also because there is no agreed definition within simulation and operations research (O.R.). This paper follows the definition of Brooks and Robinson (2001) that a conceptual model is "a software independent description of the model that is to be constructed". Conceptual modeling therefore involves deciding the way in which the virtual world of the simulation model should work, typically the entities that it contains and all the interactions, rules, and equations that determine their behavior. This is a different task to actually building the model which consists of implementing the conceptual model, usually programming it using a simulation software package. Sometimes conceptual modeling and model building can take place together, but it is still important to consider them as separate tasks since their nature and objectives are quite different.

Conceptual modeling advice often advocates keeping the model as simple as possible (e.g., Ward 1989 and Salt 1993). For example, Robinson (1994) proposed that the basic rule for what to include in a model is to use the minimum components required to achieve the project's objective, and "Model Simple - Think complicated" is one of Pidd's (2003) principles of modeling. However, definitions of level of detail and complexity are not usually provided in the literature and there are no agreed ways of measuring them.

Conceptual modeling is often thought of as a skill that improves with experience and investigating the approach followed by both experts and novices during modeling projects would provide essential foundation data for conceptual modeling research. A consensus on the modeling process is its iterative nature (e.g., Law 2007; Pidd 2003; Sánchez 2006). Therefore, studying how conceptual modeling relates to the other modeling tasks is important in improving the understanding of conceptual modeling. However, there have been very few empirical studies on conceptual modeling or the modeling process. Probably the best study of this type is that of Willemain (1995), who used a think aloud protocol whereby expert O.R. modelers spoke their thoughts during the first hour of tackling an artificial O.R. problem. Transcripts were produced from the tape recordings of the 24 sessions carried out and were coded according to which one of five topics (context, structure, realization, assessment, implementa- 


\section{Wang and Brooks}

tion) the expert was considered to be addressing. This coding was analyzed in various ways included a graph showing the coded topic at each point during the session, a box plot of the positions of the topics, and the number of alternations between each pair of topics. They found that the experts spent considerable time on all topics apart from implementation. However, the time on each topic was split into many short periods, with a lot of switching between topics especially between structure and assessment. Willemain (1994) also used a survey to obtain the views of the 12 experts who participated in the experiments. Issues covered in the survey included the nature of the experts' own modeling approaches and what they considered to be the important qualities of an effective modeler, model, modeling process and client. Recently, Willemain and Powell carried out a similar experiment to Willemain (1995) using novice modelers (Powell and Willemain 2007; Willemain and Powell 2007). They identified five main ways in which the novices fell short of what they considered to be good modeling practice, which were: over-reliance on data, taking shortcuts, insufficient use of variables and relationships, ineffective selfregulation and overuse of brainstorming.

This paper describes three studies to obtain empirical data on conceptual modeling and the modeling process. The first study followed the general approach of Willemain (1995) but collected data throughout the whole project for real simulation projects done by one expert and several groups of novices. The second study was a questionnaire of simulation experts and the third was an experiment to investigate the relationship between model size and ease of understanding.

\section{STUDY OF AN EXPERT AND NOVICE GROUPS TACKLING REAL PROJECTS}

\subsection{Results}

The objective of this study was to improve the understanding of the modeling process followed in practice by different modelers, focusing particularly on conceptual modeling. The general approach followed that of Willemain (1995) in collecting data on the topics worked on during the modeling process. However, here data was collected throughout real projects for an expert and for nine groups of novices. The study therefore differs from Willemain's in four main ways; firstly the projects are all simulation projects, secondly they are real projects, thirdly data was collected for the whole project rather than just the initial stage, fourthly groups of novices as well as an expert were followed. In fact, moving to reallife projects, looking at novices and looking at groups of modelers were all future experiments suggested by Willemain. A detailed description of the procedures and the results of the study can be found in Wang and Brooks (2007), and a brief outline is presented here.

\subsection{Projects and Data Collection}

The first project was a 10 week project carried out part time by an expert at the end of 2004 that aimed to improve the efficiency of staff usage in a call centre. The expert had about four years of modeling and simulation experience prior to the project as well as a having a Masters degree in Operational Research. The nine novice projects were studies done by groups of students at Lancaster University investigating real problems of their choosing, most of which were from the university campus (such as campus shops or the library). The students had very little previous simulation experience and these studies were the main assessment on simulation modules. Six of the projects took place in 2005 (phase 1) and three were in 2006 (phase 2). Two of the projects in 2005 were done by groups of three students on the Masters course in Operational Research. The other seven projects were done by groups of five undergraduate students.

The aim of the data collection was to identify the topics worked on during the project. The expert was asked to record the total time each week on different topics. The topic list agreed with the expert was an alternative list from the Willemain (1995) paper. Two improvements were made to this method for the 2005 novice projects: the students were asked to record data on a daily basis, and a much more detailed list of topics was provided to make it easier for the students to do the allocation and to obtain more detailed data. In 2006 the researcher (Wang Wang) sat in on most of the student group meetings, observed the students' behavior and recorded the topics herself in hourly intervals. In the subsequent analysis the novice topics were combined into our preferred topics, which are problem structuring, conceptual modeling, data collection, model coding, validation and verification, experimentation and report writing. The expert data was also allocated to these categories although this required a transformation from the original topics using the recollections of the expert.

\subsection{Results}

The analysis of the data follows some of Willemain's analysis by comparing a graphical representation of the topics over time and by calculating the percentage of time on each topic. The plots of the topics over time (Wang and Brooks 2007) showed considerable switching between topics by the expert whereas the novices had much less overlap with the pattern being closer to a linear process of completing one topic before starting the next. In particular, the expert started verification and validation much earlier in the project process.

The percentage of time spent on the topics after the re-allocation of the expert project time is shown in Figure 1. The similarity in the data between the phase 1 and phase 2 novice projects gives additional confidence in the 


\section{Wang and Brooks}

phase 1 data which was recorded by the students themselves. There are some clear differences between the expert project and the novice projects. The expert spent a much greater proportion of time on conceptual modeling and much less on data collection and report writing. The expert also spent twice as much time on verification and validation than experimentation which is quite different to the phase 2 novice projects. However, these comparisons should be treated with some caution because of the expert topic transformation involved and because the expert results are just a single project.
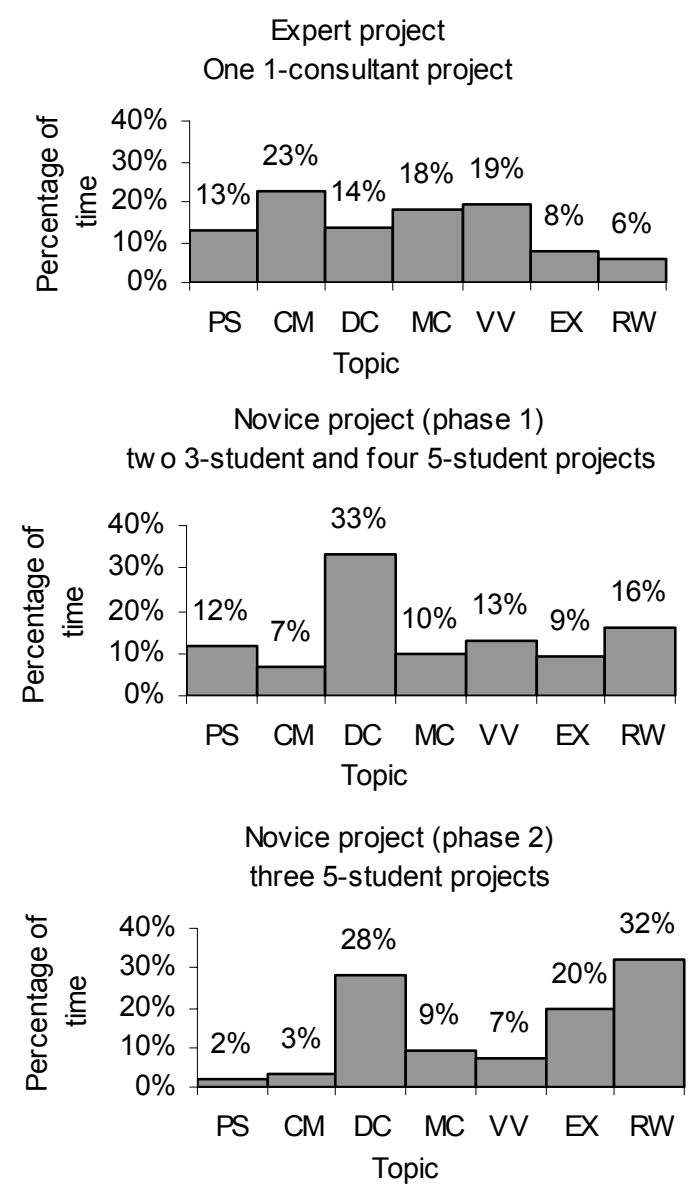

Figure 1: Weight of topics (PS = problem structuring, $\mathrm{CM}$ $=$ conceptual modeling, $\mathrm{DC}=$ data collection, $\mathrm{MC}=$ model coding, $\mathrm{VV}=$ verification and validation, $\mathrm{EX}=\mathrm{ex}$ perimentation, RW $=$ report writing) (Wang and Brooks 2007).

The differences between the expert and novices may be partly due to the nature of the projects. The novices had to collect the data themselves which the expert did not and so this explains the greater time spent on data collection by the novices. The novice projects were also simpler systems and so this may partly explain the lower proportion of time on conceptual modeling. However, additional insights were gained by interviewing the expert each week during the project, from the observations of the phase 2 novice projects and from looking at the assessment of the students' reports. The expert developed the conceptual model at the beginning of the project and documented it in a system flow diagram, which guided the construction of the computer model. The novice groups did discuss the system processes (at the start and, sometimes, during model coding), but differed from the expert in that they rarely drew a diagram (apart from sometimes drawing one at the end to include in the report) and overall devoted little time to understanding how the system actually worked.

There tended to be a lack of planning of data collection by the novice groups leading to inefficiencies in this process. Some groups didn't collect data for validation which prevented any black box validation being carried out. In the marking of the projects, verification and validation was the aspect on which the students got the lowest marks, indicating insufficient emphasis or a lack of understanding. In general, it is likely that experienced modelers have a greater appreciation than novices of the importance and benefits of both conceptual modeling and verification and validation, for example by learning from problems on previous projects.

\section{QUESTIONNAIRE SURVEY OF EXPERTS}

The main aim of the questionnaire was to obtain data from experienced modelers on their last modeling projects. The use of a survey enables data to be collected on many more projects than by following individual projects (section 2) although without quite the same level of detail regarding the process.

\subsection{Questionnaire Design and Administration}

Three initial questions were on the background of the respondent and their modeling style but the remainder of the questionnaire asked various questions on the modeling process of the most recently completed simulation project. These covered the project background, project outcome, the approach for developing the conceptual model and the way it changed during the project, the proportion of time on each modeling topic and a Gantt chart of the time spent on the topics. Overall there were 24 questions although some had several parts, with a mixture of multiple choice, likert-scale, and open-ended questions.

The questionnaire was administered to get a large sample of experienced simulation modelers. The survey took place between March 25, 2006 and May 15, 2006. Three groups were approached. Two groups were attendees at conferences, namely the UK Operational Research Society Simulation Workshop, 2006 and the Witness User Conference, 2006. Attendees were asked to fill in the questionnaire at the conferences and 40 responses were obtained. The third group was authors from the proceed- 


\section{Wang and Brooks}

ings of Winter Simulation Conference, 2005 who were emailed and were asked to complete the questionnaire online. This generated 62 responses, giving a total of 102 responses. The respondents mainly come from UK and USA, but with a few from the rest of Europe, Asia and Latin America.

\subsection{Results}

\subsubsection{Respondents' Backgrounds}

The modeling experience of the respondents ranged from one year to forty years with an average experience of twelve years. Most respondents had a significant amount of experience and so the results should reflect expert behavior.

\subsubsection{The Conceptual Model}

Figure 2 shows the problem areas of the projects. The most popular was logistics (listed as "logistics, transportation and distribution" in the survey), followed by manufacturing and military. The duration of the projects varied a lot, from short projects that lasted for only a week to long ones that took more than a year to complete. About half of the projects were completed within 6 months. Despite the diversity of the projects, the majority $(67 \%)$ of respondents stated that the project was typical of most of the projects in which they were involved, which adds more confidence that the results represent simulation modeling in general.

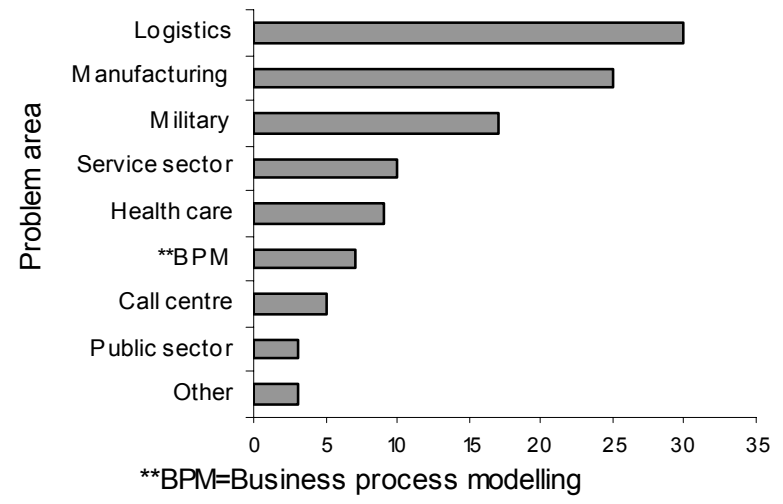

Figure 2: Problem area of the project.

Respondents were asked to select the methods they used to understand the real system and the problem. Many used several methods with the number of responses being: talk to the management $71(70 \%)$, analyze system data 70 $(69 \%)$, observe the system $54(53 \%)$, talk to system operator /server $51(50 \%)$, talk to customers $35(34 \%)$ and problem structuring method $30(29 \%)$. An open question asked the respondents to state the most difficult task on the project. The answers were then categorized with three most common being data (42 responses), technical issues (22 responses) and conceptual modeling (11 responses).

The survey asked the number of conceptual models developed, and if there was only one whether any changes were made. Most (83\%) respondents developed one conceptual model, but made some changes during the project, and only $2 \%$ developed one conceptual model with no further changes. The remaining $15 \%$ developed several conceptual models (from 2 to 8 with an average number of 4 for this group). Respondents were also asked when they developed the conceptual model in relation to model coding with the results of: before model coding $61 \%$, started before model coding, and finished while coding $33 \%$, entirely during model coding $6 \%$. It is therefore common practice for modelers to develop the conceptual model before model coding, although for a significant number there is an overlap of the conceptual modeling and model coding stages. There was some indication that those developing the conceptual model partly or entirely during model coding tended to be less experienced.

The methods used for documenting the conceptual model are shown in Table 1. A process flow diagram is the most popular, probably because it is easy to understand and to relate to the real system, especially for clients. A list of assumptions and simplifications is also widely used. Surprisingly nearly $20 \%$ used an activity cycle diagram.

Table 1: Documentation methods.

\begin{tabular}{|c|c|c|}
\hline $\begin{array}{c}\text { Documentation } \\
\text { method }\end{array}$ & Total* & $\begin{array}{c}\text { \% of } \\
\text { participant }\end{array}$ \\
\hline Process flow diagram & 64 & $63 \%$ \\
\hline $\begin{array}{l}\text { List of assumptions } \\
\text { and simplifications }\end{array}$ & 58 & $57 \%$ \\
\hline Logic diagram & 32 & $31 \%$ \\
\hline Component list & 22 & $22 \%$ \\
\hline Activity cycle diagram & 19 & $19 \%$ \\
\hline $\begin{array}{l}\text { UML (unified model- } \\
\text { ing language) }\end{array}$ & 14 & $14 \%$ \\
\hline Text description & 5 & $5 \%$ \\
\hline Visual display & 2 & $2 \%$ \\
\hline Other & 8 & $8 \%$ \\
\hline None & 5 & $5 \%$ \\
\hline Total & 229 & \\
\hline
\end{tabular}




\section{Wang and Brooks}

Table 2: Conceptual model changes during the project (from 96 respondents).

\begin{tabular}{|c|c|c|c|c|c|c|c|c|c|c|c|}
\hline \multirow[b]{2}{*}{ Modeling stage } & \multicolumn{7}{|c|}{ Conceptual model change } & \multicolumn{4}{|c|}{ Reason for change } \\
\hline & $\begin{array}{l}\text { Entities } \\
\text { addition }\end{array}$ & $\begin{array}{l}\text { Entities } \\
\text { deletion }\end{array}$ & Inter-rela & onship & $\begin{array}{l}\text { more } \\
\text { complex }\end{array}$ & simpler & $\mid \begin{array}{l}\text { Total } \\
\text { changes }\end{array}$ & \begin{tabular}{|l} 
Client's \\
requirement
\end{tabular} & $\begin{array}{l}\text { Problem } \\
\text { situation } \\
\text { changed }\end{array}$ & $\begin{array}{l}\text { Model not } \\
\text { realistic }\end{array}$ & $\begin{array}{l}\text { Better } \\
\text { information } \\
\text { about real } \\
\text { system }\end{array}$ \\
\hline Data collection & 30 & 7 & 30 & 17 & 35 & 13 & 132 & 9 & 8 & 17 & 38 \\
\hline Model coding & 35 & 12 & 37 & 14 & 38 & 13 & 149 & 16 & 11 & 19 & 34 \\
\hline Validation and verification & 18 & 3 & 25 & 10 & 25 & 12 & 93 & 11 & 4 & 18 & 21 \\
\hline Experimentation & 16 & 4 & 18 & 5 & 28 & 3 & 74 & 9 & 9 & 9 & 19 \\
\hline Total & 99 & 26 & 110 & 46 & 126 & 41 & 448 & 45 & 32 & 63 & 112 \\
\hline Percentage of responses & $22 \%$ & $6 \%$ & $25 \%$ & $10 \%$ & $28 \%$ & $9 \%$ & $100 \%$ & $18 \%$ & $13 \%$ & $25 \%$ & $44 \%$ \\
\hline
\end{tabular}

\subsubsection{Conceptual Model Changes}

The experts were asked to select the types of changes they made to the conceptual model during four other modeling stages, and the reasons for the change. Table 2 shows the results. Across the three types of change, $75 \%$ of changes made the conceptual model more complex and the other $25 \%$ made it simpler. A significant number of changes were made during all the four stages and so the respondents are willing to revisit the conceptual model throughout the project. The most common reason for a change is "better information about the real system" and the results indicate that this occurs quite often during all the stages. The reason for many more changes than reasons (448/ 112 ) is because some participants made several changes at a particular stage, but provided only one reason for all the changes.

In a separate question the respondents were asked how the structure of the final model compared to the initial conceptual model. There were $65 \%$ of responses that the final model was more complex, with $22 \%$ stating that the change was significant and $43 \%$ that it was minor. Of the $21 \%$ of responses that the model was simpler, $7 \%$ stated the change was significant and $14 \%$ that it was minor. $12 \%$ indicated that there was no change in the model and $2 \%$ did not provide an answer. Together with the results for the individual changes in Table 2, this provides some evidence of modelers tending to start with a simple model and adding detail as it becomes necessary during the project.

\subsubsection{Topic Distribution}

The respondents were asked to state the percentage of time that they spent on different topics during the project and also to draw a Gantt chart of when the topics were worked on during the project (split into 20 equal periods for the online questionnaire). Our preferred list of topics was used again here, as listed in Section 2.1 and Figure 1. Figure 3 shows that on average just over a quarter of the time was spent on model coding with a fairly even split between the other topics. This is certainly quite different to the phase 2 novice projects in Figure 1, which spent very little time on problem structuring and conceptual modeling.

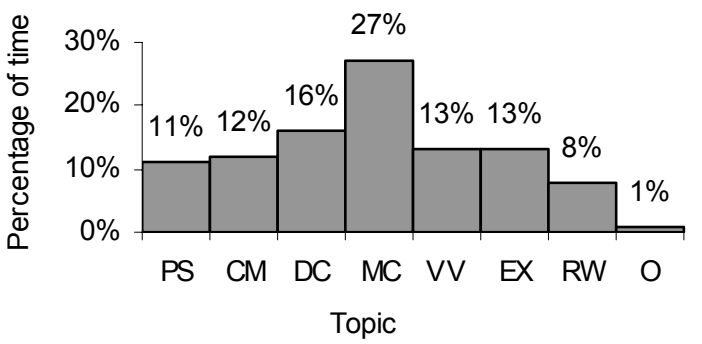

Figure 3: Proportion of time spent on each topic. Topics are as in Figure 1. $\mathrm{O}=$ others.

Figure 4 is a graphical representation of the Gantt chart results for one of the projects. If more than one topic was worked on during a time period then this is shown by the bars not being full height in the plot (a full height bar would reach the horizontal line above on the plot). For example, if a participant ticked both conceptual modeling and data collection for a given time period then this would be recorded by giving both categories a value of 0.5 .

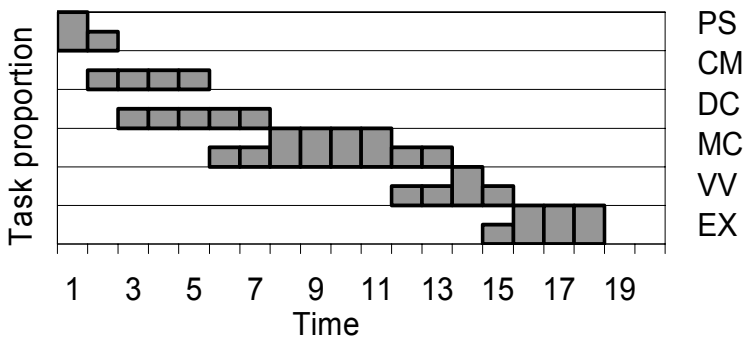

Figure 4: A typical process of a simulation project. Topics are as in Figure 1.

A comparison of all the plots showed that, in general, the topics appeared in the anticipated order with data lower down on the y-axis expected to be later, which was also confirmed by a box plot of position for all the projects. There were a lot of overlaps between the topics, in- 


\section{Wang and Brooks}

dicating that working on different tasks together is common practice. As would be expected, the most common overlaps were between successive topics in the expected order of topics.

The Gantt charts were compared visually to try and identify the main different categories of patterns. The most striking differences identified were the starting point and duration of data collection. Figure 5 shows seven positions in which data collection was initiated in the project. There were also a few unusual patterns, such as validation and experimentation being started at a very early stage of the project life cycle (Figure 6), which could be a result of using a previous model for some initial analysis.

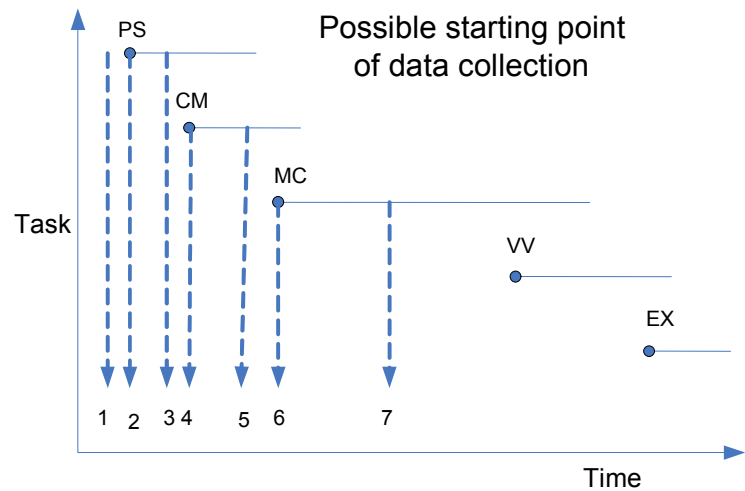

Figure 5: Time that data collected is initiated. Topics are as in Figure 1.

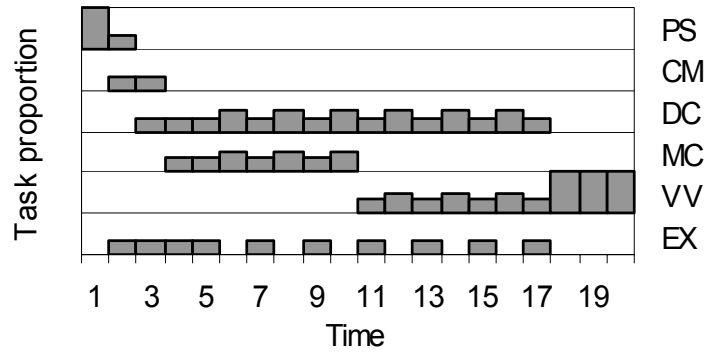

Figure 6: Simulation process that has early experimentation. Topics are as in Figure 1.

\section{EXPERIMENTS ON THE RELATIONSHIP BETWEEN MODEL CHARACTERISTICS AND MODEL PERFORMANCE}

The objective of this experiment was to investigate the relationship between model characteristics and model performance. Depending on the objectives, a project could have a combination of various performance factors such as the confidence in the model, the accuracy of the results, ease of understanding and the resources required to build and use the model (Brooks and Tobias 1996). A better understanding of the relationship between model characteristics and performance would help in the process of choosing between alternative conceptual models (Brooks 2007).

Brooks and Tobias (1996) suggested splitting model complexity into the more specific characteristics of size (the number of elements used), connectedness (the number of relationships between elements) and calculational complexity (the complexity of calculations determining the relationships). An experiment carried out previously (Brooks 1996) indicated that the difficulty in understanding the model and results is mainly caused by size and connectedness whereas build time is mainly related to calculational complexity. However, that study was a small scale experiment involving 33 Masters students.

The aim of the experiment described in this section was to investigate how the size of the model affects the understanding of the model.

\subsection{Description of the Experiment}

The experiment used four models built in WITNESS (Lanner Group Ltd., Redditch, U.K.) of a hypothetical manufacturing production line. The models were designed to differ in size (measured in the number of elements that the parts could pass through, i.e., machines and queues), but not in connectedness (measured in the relative number of routes, i.e., the number of links between elements divided by the number of elements). Model A was the simplest model and had a total of 17 different queues and machines and a total of 17 customer routes from one element to another. Models B, C and D got progressively larger with each having an extra 10 elements. Each model included 3 operators who need to operate some of the machines. Table 3 lists the size and connectedness of each model.

Table 3: Complexity of the models.

\begin{tabular}{|c|c|c|c|}
\hline & Size & Route & Connectedness \\
\hline A & 17 & 17 & 1.00 \\
\hline B & 27 & 29 & 1.07 \\
\hline C & 37 & 41 & 1.11 \\
\hline D & 47 & 53 & 1.13 \\
\hline
\end{tabular}

All four models were designed to have the same behavior and the same system bottleneck. The experimental task for the participants was to identify the bottleneck while watching the model running. The time taken to spot the bottleneck was recorded to measure the difficulty of understanding the model.

A total of 60 students from Lancaster University Management School signed up for the experiment. The majority (62\%) of participants were $\mathrm{PhD}$ students and the rest were composed of Master students (23\%) and Undergraduate students $(15 \%) .42 \%$ had some knowledge of discrete event simulation and the software they used included Witness, Simul8, Micro Saint Sharp, Promodel 


\section{Wang and Brooks}

and Arena. Figure 7 is a frequency table of the subject areas of the participants.

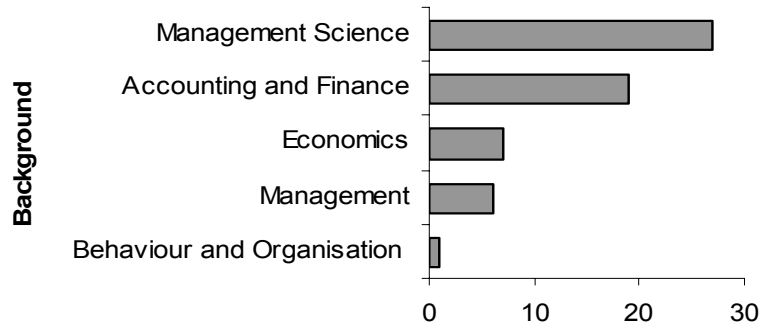

Figure 7: Subject area of the participants.

The experiment was conducted with one participant per session. A questionnaire on the participants' subject area, previous modeling experience and knowledge of operational research was filled in by the participant at the beginning of the experiment so that the researcher could try and ensure that different models were assigned randomly to those who have a similar background. The researcher then explained to the participant the experiment task and the model display, after which the participants had some time to read a document describing the model. All models were run from a steady state position $(6000$ minutes after starting the model running from being empty) at the same animation speed. There was no time limit on the experiment and the time was recorded every time an answer for the bottleneck was suggested. If the answer was incorrect, then the participant was told it was incorrect and could continue until they got the right answer or gave up. Once they correctly identified the bottleneck, the participants were asked for their reasoning in identifying it. At the end of the experiment, each participant filled in a feedback form of the experience.

\subsection{Experiment Results}

Across all the participants, the time for identifying the bottleneck ranged from 0.53 minutes to 29.59 minutes. Only 6 out of 60 participants failed to identify the bottleneck and these were excluded from the analysis of the times (the models for these six participants were one model $\mathrm{A}$, two model $\mathrm{B}$, two model $\mathrm{C}$ and one model $\mathrm{D}$ ). The average time taken to spot the correct bottleneck for each model, with the $95 \%$ confidence intervals is shown in Figure 8. As would be expected, the average time is longer for the larger models. It was hoped that the experiment would give an indication of the nature of this relationship. However, there was a large variance in the time taken for each model and a one-way ANOVA analysis of the four models did not produce a significant results at the $5 \%$ level $(p=0.09)$. Grouping the times for models $\mathrm{A}$ and $\mathrm{B}$ and comparing these with the times for models $\mathrm{C}$ and $\mathrm{D}$ does give a significant result with an independentsamples $t$ test $p$ value of 0.012 .

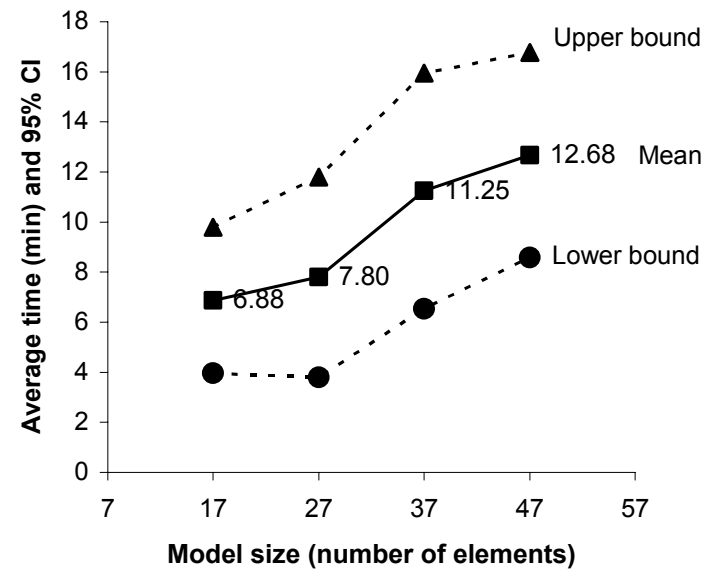

Figure 8: Average time and the $95 \%$ confidence intervals for the mean.

In finding the bottleneck, the majority $(69 \%)$ of the participants stated that the dynamic machine states were more helpful than the static statistics. Table 4 illustrates from scale 1 to 5 how easy it was for the participant to identify the bottleneck. The majority chose either 2 or 3 meaning the difficulty of the task was appropriate. In the feedback after the experiment, most of the participants considered the model description clear and the model easy to understand.

Table 4: Easiness of identifying the bottleneck.

\begin{tabular}{|l|c|c|c|c|c|}
\hline & \multicolumn{2}{|c}{ Easy } & \multicolumn{4}{c}{ } \\
\cline { 3 - 6 } & 1 & 2 & \multicolumn{3}{c|}{ Difficult } \\
\hline Frequency & 6 & 25 & 21 & 7 & 0 \\
\hline Percentage & $10 \%$ & $42 \%$ & $36 \%$ & $12 \%$ & $0 \%$ \\
\hline
\end{tabular}

\section{DISCUSSION AND FUTURE WORK}

The three pieces of empirical work described are attempts to provide empirical data to improve the understanding of conceptual modeling and the modeling process in simulation. The study following 9 student group projects revealed some useful information on novices' modeling practices. There was evidence from the data and from observing the groups of a fairly linear process with little revisiting of previous modeling topics, a lack of consideration of the conceptual model and the way the system worked, insufficient planning and not enough emphasis on verification and validation. These were all in contrast to the process followed by the expert.

The survey provided a much larger sample of projects carried out by experienced modelers and provided insights into their modeling styles. Overall, the respondents changed the model at all the different stages of the project with a tendency of increasing its complexity. There was considerable overlapping of the different modeling topics, which was also the case for the expert project in Section 2 but was quite different to the more linear style of the nov- 
ices in Section 2. This difference may be partly due to the novice projects being simpler but probably also indicates better analysis and critical evaluation by experienced modelers during the project.

The experiment comparing the model understanding and sizes found a significant modeler effect with a large variance in times for each model. This meant that even though a reasonable number of participants were obtained for the experiment, the confidence intervals for the average times were large. The results certainly indicated that larger models are harder to understand but a much larger sample would be needed to identify the pattern of this relationship.

Obtaining empirical data relevant for conceptual modeling is difficult and much more work needs to be done. Future work could include following a real smallscale consultancy project, and then repeating it in a classroom with novices. This would enable a better comparison of styles although finding a suitable project could be difficult. For better interpretation and generalisation of the relationships between model characteristics and model performance, similar experiments with more models, alternative applications and bigger sample sizes would be useful, although again there are practical problems. In addition, there is a need to investigate the relationship between other aspects of complexity and model performance.

A better understanding of the modeling approach used by experts and the differences compared to novices can provide a strong basis for conceptual modeling research and can also provide useful information for teaching novices. For example, it could be a useful practice to ask novices to compute a Gantt chart to monitor their work progress as a supplement to the project report.

\section{ACKNOWLEDGMENTS}

The authors wish to sincerely thank all who participated in the projects, the survey, and the experiment.

\section{REFERENCES}

Brooks, R. J. 1996. A framework for choosing the best model in mathematical modelling and simulation. $\mathrm{PhD}$ Thesis, Faculty of Engineering, University of Birmingham, Birmingham.

Brooks, R. J. 2007. Conceptual modelling: framework, principles, and future research. Working paper no. 2007/011, Lancaster University Management School, Lancaster, U.K..

Brooks R. J., and S. Robinson. 2001. Simulation, with Inventory Control (author C. Lewis), Operational Research Series. Basingstoke: Palgrave.

Brooks, R. J., and A. M. Tobias. 1996. Choosing the best model: Level of detail, complexity, and model per- formance. Mathematical and Computer Modeling 24(4): 1-14.

Law, A. M. 1991. Simulation-models level of detail determines effectiveness. Industrial Engineering 23(10): 16-18.

Law, A. M. 2007. Simulation Modeling and Analysis. 4th edition. New York: McGraw-Hill.

Pidd, M. 2003. Tools for Thinking, Modelling in Management Science. Chichester: John Wiley and Sons.

Powell, S. G., and T. R. Willemain. 2007. How novices formulate models. Part I: qualitative insights and implications for teaching. Journal of Operational Research Society in press.

Robinson, S. 1994. Simulation projects - Building the right conceptual-model. Industrial Engineering 26(9): 34-36.

Robinson, S. 2004. Simulation: the Practice of model Development and Use. Chichester: John Wiley and Sons.

Salt, J. D. 1993. Simulation should be easy and fun! In proceedings of 1993 Winter Simulation Conference, ed. G.W. Evans, M. Mollaghasemi, E.C. Russell and W.E. Biles, 1-5. New York: Institute of Electrical and Electronics Engineers. Inc.

Sánchez, P. J. 2006. As simple as possible, but no simpler: A gentle introduction to simulation modeling. In proceedings of 2006 Winter Simulation Conference, ed. L.F. Perrone, F.P. Wieland, J. Liu, B.G. Lawson, D.M. Nicol, and R.M. Fujimoto, 2-11.

Shannon R. E. 1975. Systems Simulation: The Art and Science, New York: John Wiley.

Wang, W., and R. J. Brooks. 2007. Improving the understanding of conceptual modelling. Journal of Simulation in press.

Ward, S. C. 1989. Arguments for constructively simple models. Operational Research Society 40(2): 141153.

Willemain, T. R. 1994. Insights on modeling from a dozen experts. Operations Research 42(2).

Willemain, T. R. 1995. Model formulation: What experts think about and when. Operations Research 43(6).

Willemain, T. R., and S. G. Powell. 2007. How novices formulate models. Part II: a quantitative description of behaviour. Journal of the Operational Research Society in press.

\section{AUTHOR BIOGRAPHIES}

WANG WANG is a PhD student in the Department of Management Science at Lancaster University. She completed her first degree in Management Science (Hons) at Lancaster University at 2003. Email to $<w$.wang3elancaster.ac.uk>.

ROGER J. BROOKS is a lecturer in the Management Science department at Lancaster University. He received 


\section{Wang and Brooks}

a PhD and MSc in Operational Research from Birmingham University and a B.A. (Hons) degree in mathematics from Oxford University. He is co-author of a textbook on simulation. His areas of interest include conceptual modeling, Boolean networks and agent-based simulation. His e-mail is <roger.brooks l lancaster.ac.uk> and his web page can be found via the University website: <ww. lums.lancs.ac.uk>. 